\title{
The Acute Effects of Pre-Conditioning Activities with a Weighted Vest on Subsequent Linear Sprint and Change of Direction Performance in Physical Education Students
}

\author{
Celil Kaçoğlu ${ }^{1}$ \\ Izzet Kirkaya ${ }^{2 \times}$
}

'Eskişehir Technical University, Sport Sciences Faculty, Department of Coaching Education, Eskişehir, Turkey. Email:chacoglu@eskisehir.edu.trTel: +905064017872

${ }^{2}$ Oozgat Bozok University, School of Physical Education and Sports, Yozgat, Turkey.

Emailizizzet.kirkaya@yobu.edu.trTel: +905554912161

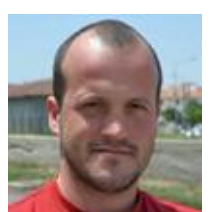

( Corresponding Author)

\section{Abstract}

The aim of this study was to determine the effects of a post activation potential application response in sprinting and change of direction performance. Fifteen physically active, healthy men (age 23.5 \pm 2.0 years, height $175.0 \pm 7.6 \mathrm{~cm}$, body weight $72.2 \pm 8.5 \mathrm{~kg}$, body fat $15.7 \pm 2.9 \%$ ) voluntarily participated in this study. Cross sectional research design with a single group $(n=15)$ and repeated tests was used and applicants participated in sprint and pro-agility tests 8 minutes after the pre-conditioning (PC) with weight wests that corresponded $5 \%$ and $10 \%$ of their body weights in different days without pre-conditioning. For PC, 8 minutes before for each $30 \mathrm{~m}$ sprint and pro-agility tests, they did a resisted running with a weight vest corresponding $5 \%$ and $10 \%$ of their body weights. Analysis shows that, sprint after PC activity as resistance running with $\% 5$ and $\% 10$ of body weight $(\mathrm{p}<0.05)$ and agility $(\mathrm{p}<0.05)$ data showed statistically significant difference. According to the results of Bonferroni post-hoc correction, 30m sprint test times, which were performed 8 minutes after the PC which includes a $30 \mathrm{~m}$ sprint with weight vests corresponding to $5 \%$ of their body weight, showed an increase from control test times, $4.34 \pm 0.23$ seconds to $4.40 \pm 0.24$ seconds respectively. This 0.07 second increase is statistically significant $(\mathrm{p}<0.05)$. In our study, PAP effect being observed as negative, might be related with the lower body weight percentages of resisted running exercise which was selected for PAP.

Keywords: Pre-conditioning contraction, Post activation potentiation, Weighted vest, Sprint, Change of direction, Agility.

Citation | Celil Kaçoğlu; İzzet Kirkaya (2020). The Acute Effects of Pre-Conditioning Activities with a Weighted Vest on Subsequent Linear Sprint and Change of Direction Performance in Physical Education Students. Asian Journal of Education and Training, 6(3): 341-346.

History:

Received: 28 February 2020

Revised: 7 April 2020

Accepted: 12 May 2020

Published: 4 June 2020

Licensed: This work is licensed under a Creative Commons

Attribution 3.0 License $($ (c))

Publisher: Asian Online Journal Publishing Group
Acknowledgement: Authors would like to thank their student volunteers from Eskişehir Technical University and Student Rıdvan Uzar, for a valuable contribution and dedication to the current study.

Funding: This research article is funded by Eskisehir Technical University. Competing Interests: The authors declare that they have no conflict of interests.

Transparency: The authors confirm that the manuscript is an honest, Transparency: The authors confirm that the manuscript is an honest,
accurate, and transparent account of the study was reported; that no vital accurate, and transparent account of the study was reported; that no vital
features of the study have been omitted; and that any discrepancies from the features of the study have been omitted
study as planned have been explained.

Ethical: This study follows all ethical practices during writing.

\section{Contents}

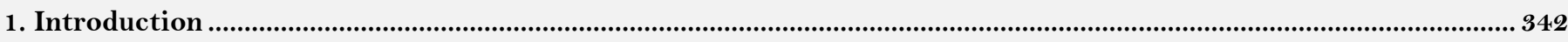

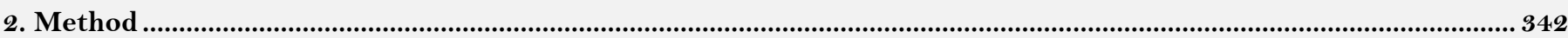

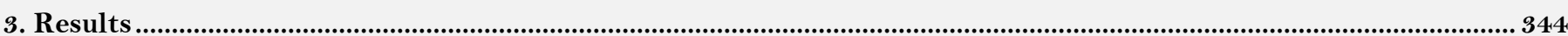

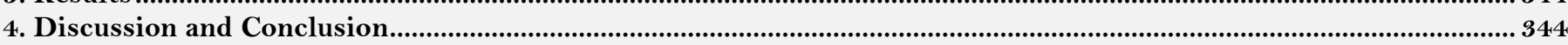

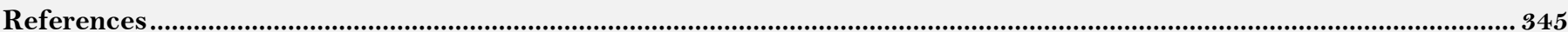




\section{Contribution of this paper to the literature}

This research aims to reveal post activation potential (PAP). A wide variety of methods are seen in the literature to mediate the performance effects of preconditioning activities. It is thought that the preconditioning effects of resistance applications such as weight vest will contribute positively to the literature. It constitutes a resource for athletes, coaches and sports experts on PAP phenomenon.

\section{Introduction}

Acute practices that will improve performance for all sports branches are seen here. Especially branches which include anaerobic performances, sprint and force give more attention to this approach (Yilmaz, 2019). A number of training and recovery strategies are used to enable athletes to perform optimally on the day of training or competition. Performance can be greatly enhanced by some specific pre-conditioning (PC) methods. Some of these performance enhancement strategies are; post activation potential (PAP), ischemic pre-conditioning, warmup protocol, passive heat application, morning exercise session or hormonal preparation (Kilduff, Finn, Baker, Cook, \& West, 2013).

Among these approaches, especially PAP application maximizes the acute strength increase of athletes (Lorenz, 2011). PAP application affects muscle strength rate increase and strength development rate (RFD) positively, thanks to previously performed muscle activation. Stimulation of nervous system under load, increases contraction functions more (Judge, 2009; Mitchell \& Sale, 2011). Applicability of PAP is mostly concentrated on exercises which use strength. Because PAP, which occurs after maximum exercise, could maintain the acute PC stimulus which increases muscle strength output for a few minutes (Seitz \& Haff, 2016). The phenomenon caused by PC activities performed maximally or close maximally during next exercise is called force-power potentiation complex as well (Stone, Sands, Pierce, Ramsey, \& Haff, 2008). Muscle performance that will occur after PC is directly related to the balance between fatigue and potentiation (Rassier \& Macintosh, 2000). Increased muscle performance depends on fatigue dropping, while potentiation is peaking. If the level between the two conditions is the same of fatigue level is higher, an increase in performance will not be observed. Again, the balance between these two conditions depends on the force-power potentiation complex and individual characteristics (Tillin \& Bishop, 2009). In studies studying the characteristics of individuals, it has been seen that potentization levels of strong individuals are higher than those of weak individuals (Ruben et al., 2010; Seitz, de Villarreal, \& Haff, 2014). In addition, after intensive PC, strong individuals exhibited the PAP affect much faster than their weak counterparts (Jo, Judelson, Brown, Coburn, \& Dabbs, 2010; Seitz et al., 2014). Therefore this study aims to try to increase the potential impact of PAP, as a PC strategy, to increase strength generation rate of volunteering participants with training experience.

\subsection{Research Method}

Fifteen physically active, healthy physical education students (age $23.5 \pm 2.0$ years, height $175.0 \pm 7.6 \mathrm{~cm}$, body weight $72.2 \pm 8.5 \mathrm{~kg}$, body fat percentage $15.7 \pm 2.9 \%$ ) with training experience in sprint and change direction, voluntarily participated in this study. The purpose of this study was explained to the participant in detail, and it was stated that they need to use maximum effort in all the tests and applications they participated during this research and they were also encouraged verbally during the tests. Details of the research were explained to all participants and they were told they were free to leave the research at any stage.

During the research Helsinki Declaration was followed and this study was approved by Eskişehir Osmangazi University Clinical Research Ethics Committee (Date: 14 February 22019; Decision no: 25; Document Verification address:https://ebysnetm.ogu.edu.tr/Home/Dogrulama/71ff8503-2717-401c-829a-db81fa48a8a9). In this study, a cross sectional research design with a single group $(n=15)$ and repetitive tests was used and applicants participated in sprint and pro agility tests 8 minutes after the PC with weight wests that corresponded $5 \%$ and $10 \%$ of their body weights in different days without PC.

\section{Method}

All participants joined the test and practices one on one for trial and adaptation. Approximately 10 days after trial evaluations, all volunteers came for sprint and agility tests, one pre-test and two experimental evaluations (resistance running with a weight vest that corresponds $5 \%$ and $10 \%$ of their body weight), on 5 different days. Height, weight and body fat percentage measurements of the participants were taken as they came to the laboratory for the trial and adaptation sessions. On the pre-test day, $30 \mathrm{~m}$ sprint test was performed, and after an at least 15 minute passive resting break, agility test was started. Evaluations were on the tartan track of Eskişehir Technical University, Faculty of Sport Sciences, indoor athletics track. Before all tests and evaluations, approximately 10 minutes of mid-tempo (self-selected) flat race, followed by submaximal stretches and standard warm-up with dynamic contractions (concentric, plyometric calisthenics and various jumps) were performed. The process of the test is shown in Figure 1.

\subsection{Practice of Pre-Conditioning Activity}

Before the pre-test, participants were given a certain amount of time for standard warm-up practices and a reasonable time was provided for stretching after the warm-up period. And after the warm-up period, PC was started. For PC, 8 minutes before for each 30 meter sprint and pro agility tests, they did a resistance running with weight vests corresponding $5 \%$ and $10 \%$ of their body weights once. Since the optimal resting time is 8 to 12 minutes for optimal potentiation after PC, (Bevan, Owen, Cunningham, Kingsley, \& Kilduff, 2009; Gouvêa, Fernandes, César, Silva, \& Gomes, 2013) after this 1 repeat maximal resistance running and agility, they were given an 8 minute passive resting time and then again started on 1 repeat 30 meter sprint and agility tests. A minimum of 48 hours resting period was provided between the sprint rest day and the agility tests. With that, they were participated in evaluations 5 times in total, once for the pre-test, and twice for each agility and sprint tests 
which were performed after the PC, executed with mentioned $\% 5$ and $10 \%$ body weight corresponding weight vests and as resistance running Figure 1.

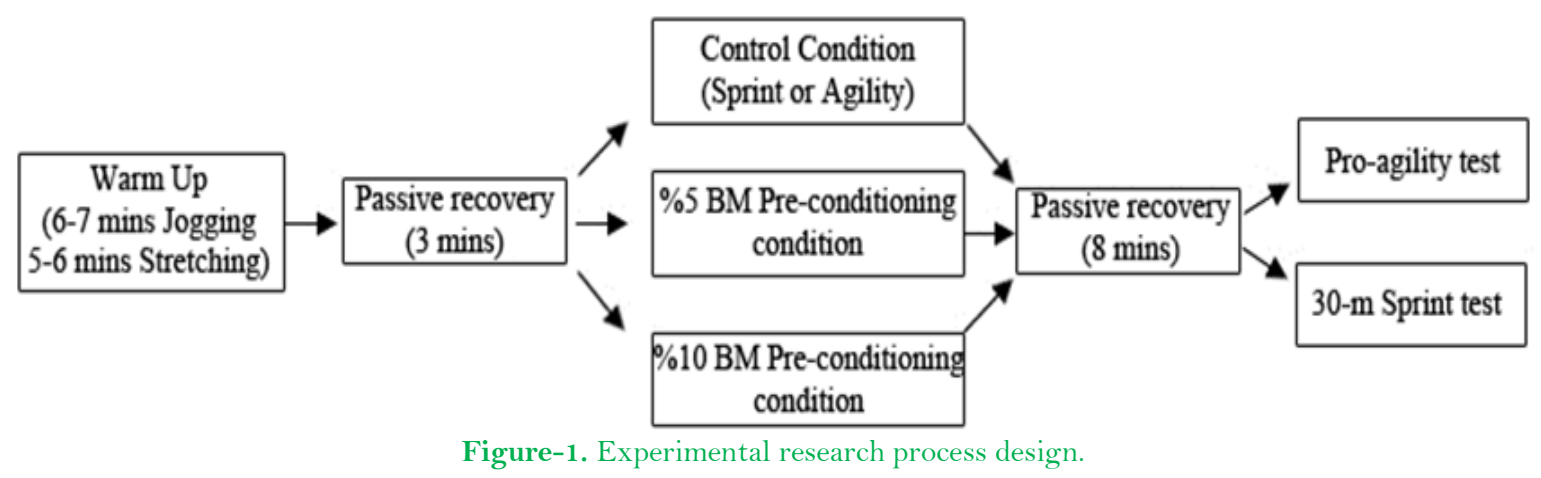

\subsection{Performance Measurements}

\subsection{1. $30 \mathrm{~m}$ Sprint Test}

Sprint acceleration performances of participants were evaluated with $30 \mathrm{~m}$ sprint run tests. A photocell and its reflectors, which were approximately 2 meters apart from the starting line and the $30^{\text {th }}$ meter, were placed mutually Figure 2. Sprint tests were tested on an indoor athletic track (Smartspeed, Fusion Sport, Australia), with an electronic stopwatch system which started the time as the participant ran through the first sensor and recorded the participants' run time between the 0 and 30 meter sensors with an accuracy of $0.01 \mathrm{~s}$. Sprint run started at the moment determined by the participant within the area determined by a line which was $1 \mathrm{~m}$ behind the starting point and at the moment when the participant hit the photocell on the starting line with a forward movement without a backwards move and completed when they hit the 30m line sensor. The participants were given the necessary information that they needed to run mentioned 30m distance as quickly as possible and at a maximum speed before each test. Before the sprint tests, the participants did a standard 10 minute warm-up with low-intensity acceleration runs and various incremental running exercises (Woolford, Polglaze, Rowsell, \& Spencer, 2013). A maximal $30 \mathrm{~m}$ run was executed 8 minutes after the PCs performed with $\% 5$ and $\% 10$ body weight corresponding weight vests and as resistance running and recorded for level analysis.

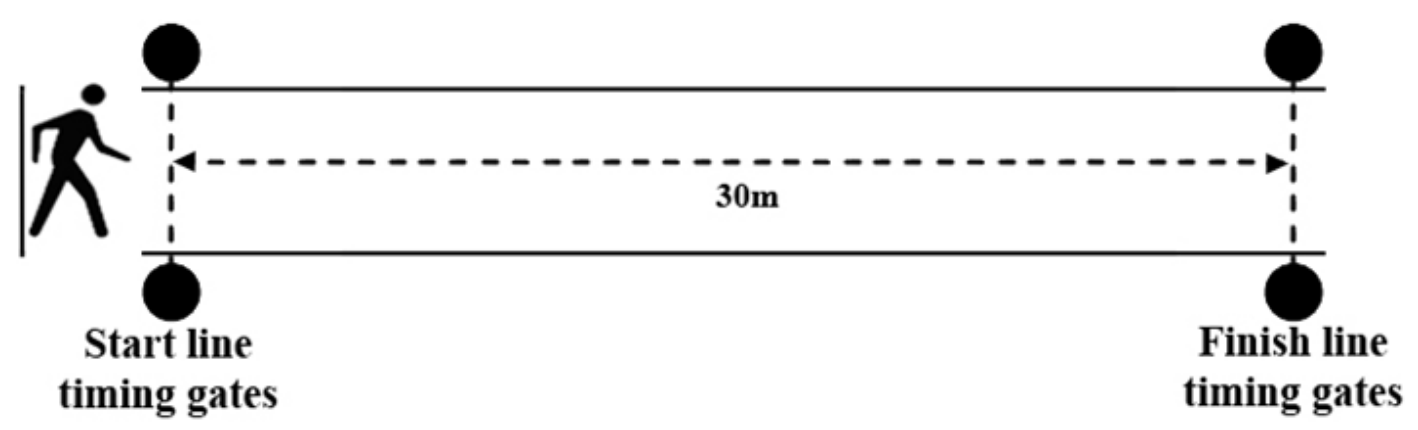

Figure-2. Graphic representation of 30m sprint running test.

\subsection{Pro-Agility Agility Test}

Directional running performances of the participants were evaluated with the pro-agility agility test. Participants started this tests from a stationary position in between a photocell chronometer located in between two parallel lines with ten yards between them and its reflector (Smartspeed, Fusion Sport, Australia) at the time and direction of their choosing. After starting the test, participants first ran until the first line for 5 yards then after touching the line, rotating for 180 degrees, they ran 10 yards to touch the other line, then again with a $180^{\circ}$ rotation, they ran 5 yards and passed the photocell and completed the test by running a total of 20 yards Figure 3. If a participant returned without touching the line, their run time was not recorded and their result was obtained by repeating the evaluation after a break of at least one day (Karacabey, 2013; Lockie, Jeffriess, Schultz, \& Callaghan, 2012).

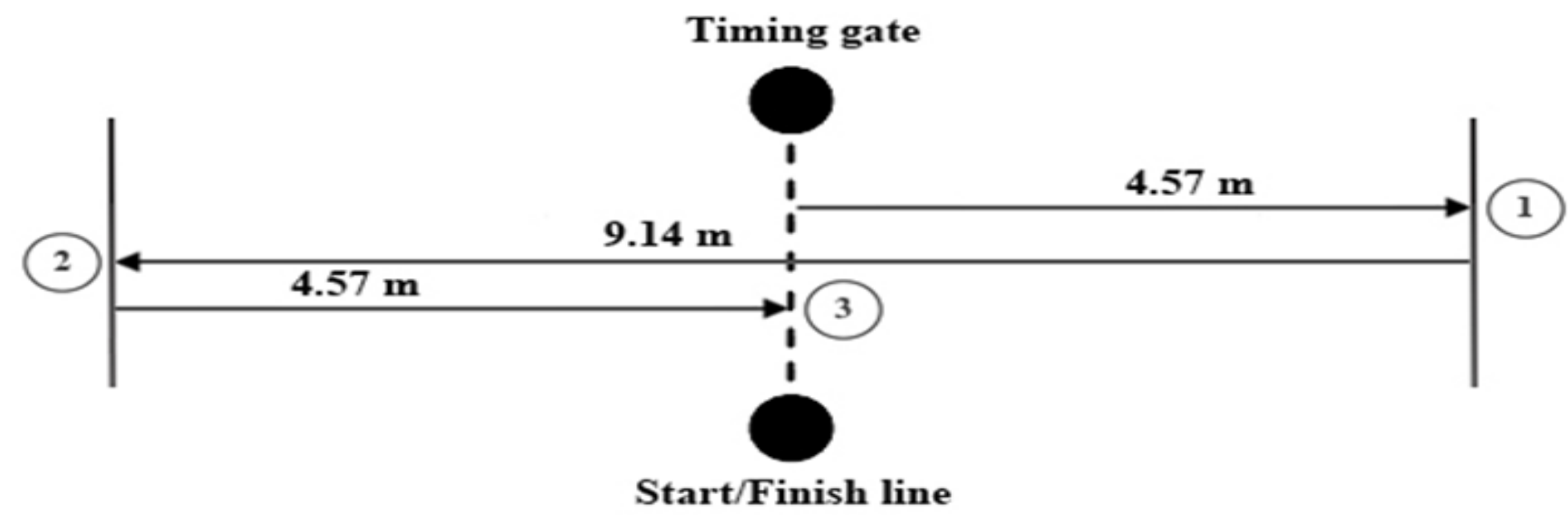

Figure-3. Graphic representation of pro-agility agility test (in this example, right side is shown as the first preference of direction). Source: Re-illustrated from Lockie et al. (2012). 


\subsection{Statistical Analysis}

Data are given as average and standard deviation. In the normality analysis of the data, with Shapiro-Wilk test $(p>0,05)$ data distributed normally and $Q-Q$ plot distribution showed that there were no extreme values in the test data. To determine whether there was a difference between sprint and agility performances that were performed after PC with weight vests that correspond to $\% 5$ and $\% 10$ body weight and the pre-test values of these performances, one-way variance analysis was used in repeated evaluations. The significance level was determined as $\mathrm{p}<0.05$ in all statistical tests. If the $\mathrm{F}$ value of the analysis turns out to be significant, post-hoc comparison was used to determine which values differ. Mauchly's Sphericity test assumption was proved for sprint values $\chi 2(2)=0.149, \mathrm{p}$ $=0.928$ but not for agility $\chi^{2}(2)=6.140, \mathrm{p}=0.046$. Therefore, Greenhouse-Gaisser correction was used for the analysis of agility tests $(\varepsilon=0.989)$. SPSS packaged software was used for data analysis (Version 20.0; SPSS Inc., Chicago, IL).

\section{Results}

According to the analysis results, sprint after PC activity as resistance running with $\% 5$ and \%10 of body weight $\left[F(2,28)=20.616, p=0.000\right.$, partial $\left.\eta^{2}=0.596\right]$ and agility $[F(1.453,20.342)=4.539, p=0.033$, influence quantity $\left.\eta^{2}=0.245\right]$ data showed statistically significant difference.

According to the results of Bonferroni post-hoc correction, 30m sprint test times, which were performed 8 minutes after the PC which includes a $30 \mathrm{~m}$ sprint with weight vests corresponding to $5 \%$ of their body weight, showed an increase from control test times, 4.34 \pm 0.23 seconds to 4.40 \pm 0.24 seconds respectively. This 0.07 second increase is statistically significant (\%95 IC, $-0.14,0.01, \mathrm{p}<0.05)$. 30m sprint test times, which were performed 8 minutes after the PC which includes weight vests corresponding to $10 \%$ of their body weight, showed an increase from $30 \mathrm{~m}$ sprint test times, which were performed 8 minutes after the $\mathrm{PC}$ which includes weight vests corresponding to $5 \%$ of their body weight, from $4.40 \pm 0.24$ seconds to $4.50 \pm 0.29$ seconds respectively. This 0.10 second increase is statistically significant $(\% 95 \mathrm{IC},-0.18,0.14, \mathrm{p}<0.05)$. 30m sprint test times, which were performed 8 minutes after the PC which includes a 30m sprint with weight vests corresponding to $10 \%$ of their body weight, showed an increase from control test times, $4.34 \pm 0.23$ seconds to $4.50 \pm 0.29$ seconds respectively. This 0.17 second increase is statistically significant (\%95 IC, $-0.24,0.01, \mathrm{p}<0.05$ ).

According to the results of Bonferroni post-hoc correction, the pro-agility agility performance times which were performed 8 minutes after the PC which includes a pro-agility agility test with weight vests corresponding to $5 \%$ of their body weight, showed an increase from control test times, $5.18 \pm 0.25$ seconds to $5.18 \pm 0.36$ seconds respectively. This 0.01 second increase is not statistically significant (\%95 IC, -0.21, 0.21, p >0.05). Pro-agility agility test performance times, which were performed 8 minutes after the pro-agility based PC which includes weight vests corresponding to $10 \%$ of their body weight, showed an increase from the pro-agility test performance times, which were performed 8 minutes after the $\mathrm{PC}$ which includes weight vests corresponding to $5 \%$ of their body weight, from $5.18 \pm 0.36$ seconds to $5.35 \pm 0.27$ seconds respectively. This 0.17 second increase is not statistically significant (\%95 IC, -0.37, 0.30, p>0.05). The pro-agility agility performance times which were performed 8 minutes after the pro-agility based PC which includes weight vests corresponding to $10 \%$ of their body weight, showed an increase from control test times, $5.18 \pm 0.25$ seconds to $5.35 \pm 0.27$ seconds respectively. This 0.07 second increase is statistically significant (\%95 IC, $-0.28,-0.61, \mathrm{p}<0.05)$ Figure 4.

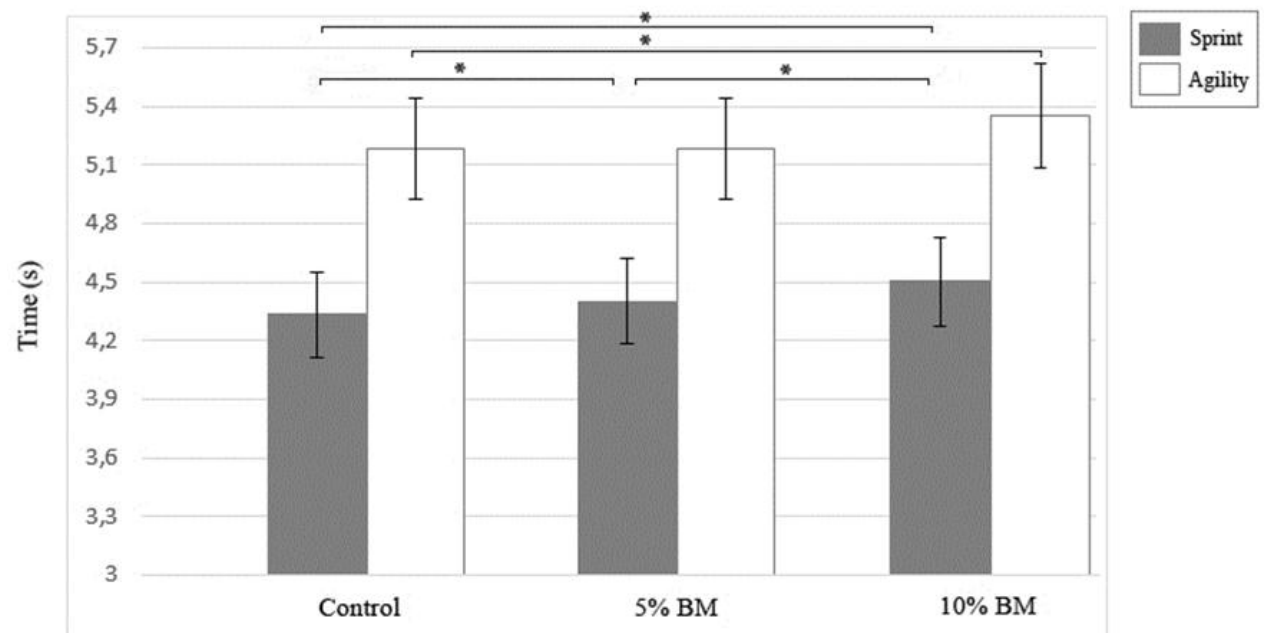

Figure-4. 30m Sprint and pro-agility agility performance averages of participants $(* \mathrm{p}<0.05)$.

\section{Discussion and Conclusion}

Dominant view in literature says, if the PAP effect is desired to be seen for sprint, activities biomechanically similar to sprint should be performed (Crewther et al., 2011; Whelan, O'Regan, \& Harrison, 2014). In this part of the study, we will evaluate the effects of PAP methods created to improve sprint times in literature.

Dello Iacono and Seitz (2018) used barbell hip thrust to create PAP effect in their study. In the study which they performed using $85 \%$ of $1 \mathrm{RM}$, they wished to observe the performance for sprint distances of 5,10 and 20 meters. 5 and $10 \mathrm{~m}$ sprints performed $15 \mathrm{~s}$ after PAP, showed deteriorations in performance, while sprints performed after 4 and 8 minutes after PAP, showed improved performances. Compared with our research, we can say that barbell hip thrust exercise gives relatively more effective results in creating PAP. Mcbride, Nimphius, and Erickson (2005) stated that the weighted counter movement jump exercise with $30 \%$ of $1 \mathrm{RM}$, has a positive PAP effect on the 40m sprint performance. In another study examining the PAP effects after a resistance running performed by using $10 \%, 20 \%$ and $30 \%$ of their body weights, achieved an approximate $1.2 \%$ improvement in a $35 \mathrm{~m}$ sprint performance after PAP in which a $10 \%$ of body weight resistance was used (Smith et al., 2014). Also, there 
was no change in 10 and $20 \mathrm{~m}$ sprints performed $15 \mathrm{~s}$ after a plyometric PAP using $10 \%$ of body weight. However, an improvement was observed in 10 and 20m sprints performed after 4 and 8 minutes (Turner, Bellhouse, Kilduff, \& Russell, 2015). This result shows conflictions with our research Figure 4. Winwood, Posthumus, Cronin, and Keogh (2016) tried to observe the effects of PAP after performing resistance sprint using $75 \%$ of body weight at $7.5 \mathrm{~m}$ and $150 \%$ of body weight at $15 \mathrm{~m}$. Fifteen-meter sprint times performed after 4,8 and 12 minutes were negatively affected. In another study involving resistance running, no changes were seen in 5 and $10 \mathrm{~m}$ sprint times performed after 2, 4, 6, 8 and 10 minutes and with $25-30 \%$ resistance in $10 \mathrm{~m}$ with 3 repetitions (Whelan et al., 2014).

In studies examining the PAP effect on resistance running so far, various different methods have been used. Naturally, due to this, different methods have been tried and studies were not evaluated within the same pool. PAP studies aiming to improve sprint times, mostly focused on back squat, power clean, plyometric exercises and resistance running. However, despite 4 different PAP approaches emerging, a clear result is yet to be acquired (Healy \& Comyns, 2017).

In a study with young male soccer players, 1 RM-\%80x 5 repetitive back squat formula was used for the PAP effect and they obtained positive results in T-drill agility tests which were performed after 8 minutes (Petisco et al., 2019). Okuno et al. (2013) performed PAP with back half squat exercise with $50 \%$ of $1 \mathrm{RM}$ in $5,70 \%$ of $1 \mathrm{RM}$ in 3 and $90 \%$ of $1 \mathrm{RM}$ in $5 * 1$ repetitions. They performed an agility test after 5 minutes and obtained a $-1,37 \%$ time change. Sole, Moir, Davis, and Witmer (2013) used back squat exercise for PAP effect in their studies. 4, 8 and 12 minutes after back squat performed repeatedly with $50 \%$ of $1 \mathrm{RM} 5,60 \%$ of $1 \mathrm{RM} 3$ and $90 \%$ of $1 \mathrm{RM} 3$ times, they performed a $10 \mathrm{~m}$ shuttle run. And they achieved a better result of $-2,27 \%$ in the shuttle run which was performed 12 minutes after. Comparing the agility results with the research results, we can see that the obtained results are not similar. One of the important factors in direction change runs is to produce vertical and horizontal forces in the stopping phase. An effective deceleration could be achieved by this. An exercise performed with weights, such as back squat, may be providing the needed vertical strength during 180 degree turns. Being able to manipulate these forces in stopping phase, may provide rapid direction changes (Lockie, Lazar, Davis, \& Moreno, 2018). Okuno et al. (2013) on the other hand, stated that technique also has great influence in direction changing agility runs as much as speed. They also mentioned that bilateral Pc activities such as back squat, will have a positive effect on direction changing agility runs.

If we reconsider the PAP studies targeting performance improvements in sprint and direction change agility running once again, we can say that an increase in performance is generally observed when the running is performed after 4 to 12 minutes. And it is considered appropriate to perform PAP exercises within the range of 3-6 sets and 5-6 repetitions instead of 1 rep and 1 set (Duthie, Young, \& Aitken, 2002). Another study states that it is appropriate to perform sprint runs after approximately 4 to 8 minutes of recovery to see the PAP effects of exercises such as back squat and power clean performed with 90\% intensity (Healy \& Comyns, 2017). In our study, PAP effect being observed as negative, might be related the low $\%$ intensity of resistance running exercise which was selected for PAP.

\section{References}

Bevan, H. R., Owen, N. J., Cunningham, D. J., Kingsley, M. I., \& Kilduff, L. P. (2009). Complex training in professional rugby players: Influence of recovery time on upper-body power output. The Journal of Strength \& Conditioning Research, 23(6), 1780-1785.Available at: https://doi.org/10.1519/jsc.0bo13e3181b3f269.

Crewther, B. T., Kilduff, L. P., Cook, C. J., Middleton, M. K., Bunce, P. J., \& Yang, G. Z. (2011). The acute potentiating effects of back squats on athlete performance. The Journal of Strength $\mathcal{F}^{\circ}$ Conditioning Research, 25(12), 3319-3325.Available at: https://dx.doi.org/10.1519/JSC.obo13e318215f560.

Dello Iacono, A., \& Seitz, L. B. (2018). Hip thrust-based PAP effects on sprint performance of soccer players: Heavy-loaded versus optimumpower development protocols. Journal of Sports Sciences, 36(20), 2375-2382.Available at: https://doi.org/10.1080/02640414.2018.1458400.

Duthie, G. M., Young, W. B., \& Aitken, D. A. (2002). The acute effects of heavy loads on jump squat performance: An evaluation of the complex and contrast methods of power development. The Journal of Strength Eं Conditioning Research, 16(4), 530-538.Available at: https://doi.org/10.1519/00124278-200211000-00007.

Gouvêa, A. L., Fernandes, I. A., César, E. P., Silva, W. A. B., \& Gomes, P. S. C. (2013). The effects of rest intervals on jumping performance: A meta-analysis on post-activation potentiation studies. Journal of Sports Sciences, 31(5), 459-467.Available at: https://doi.org/10.1080/02640414.2012.738924.

Healy, R., \& Comyns, T. M. (2017). The application of postactivation potentiation methods to improve sprint speed. Strength and Conditioning Journal, 39(1), 1-9.Available at: https://doi.org/10.1519/ssc.0000000000000276.

Jo, E., Judelson, D. A., Brown, L. E., Coburn, J. W., \& Dabbs, N. C. (2010). Influence of recovery duration after a potentiating stimulus on muscular power in recreationally trained individuals. The Journal of Strength \& Conditioning Research, 24(2), 343-347.Available at: https://doi.org/10.1519/jsc.0bo13e3181cc22a4.

Judge, L. W. (2009). The application of postactivation potentiation to the track and field thrower. Strength E Conditioning Journal, 31(3), 3436.Available at: https://doi.org/10.1519/ssc.0bo13e3181a62960.

Karacabey, K. (2013). Sport performance and agility tests. Journal of Human Sciences, $10(1)$, 1693-1704.

Kilduff, L. P., Finn, C. V., Baker, J. S., Cook, C. J., \& West, D. J. (2013). Preconditioning strategies to enhance physical performance on the day of competition. International Journal of Sports Physiology and Performance, 8(6), 677-681.Available at: https://doi.org/10.1123/ijspp.8.6.677.

Lockie, R. G., Jeffriess, M. D., Schultz, A. B., \& Callaghan, S. J. (2012). Relationship between absolute and relative power with linear and change-of-direction speed in junior American football players from Australia. Journal of Australian Strength and Conditioning, 20(4), $4-12$.

Lockie, R. G., Lazar, A., Davis, D. L., \& Moreno, M. R. (2018). Effects of postactivation potentiation on linear and change-of-direction speed: analysis of the current literature and applications for the strength and conditioning coach. Strength \& Conditioning Journal, 4O(1), 75-91.Available at: https://doi.org/10.1519/ssc.0000000000000277.

Lorenz, D. (2011). Postactivation potentiation: An introduction. International Journal of Sports Physical Therapy, 6(3), 234-240.

Mcbride, J. M., Nimphius, S., \& Erickson, T. M. (2005). The acute effects of heavy-load squats and loaded countermovement jumps on sprint performance. Journal of Strength and Conditioning Research, 19(4), 893-897.Available at: https://dx.doi.org/10.1519/00124278$200511000-00029$.

Mitchell, C. J., \& Sale, D. G. (2011). Enhancement of jump performance after a 5-RM squat is associated with postactivation potentiation. European Journal of Applied Physiology, 11 1(8), 1957-1963.Available at: https://doi.org/10.1007/s0042 1-010-1823-x. 
Okuno, N. M., Tricoli, V., Silva, S. B., Bertuzzi, R., Moreira, A., \& Kiss, M. A. (2013). Postactivation potentiation on repeated-sprint ability in elite handball players. The Journal of Strength \& Conditioning Research, 27(3), 662-668.Available at: https://doi.org/10.1519/jsc.obo13e31825bb582.

Petisco, C., Ramirez-Campillo, R., Hernández, D., Gonzalo-Skok, O., Nakamura, F., \& Sanchez-Sanchez, J. (2019). Post-activation potentiation: Effects of different conditioning intensities on measures of physical fitness in male young professional soccer players. Frontiers in Psychology, 10, 1167-1167.Available at: https://doi.org/10.3389/fpsyg.2019.01167.

Rassier, D., \& Macintosh, B. (2000). Coexistence of potentiation and fatigue in skeletal muscle. Brazilian Journal of Medical and Biological Research, 33(5), 499-508.Available at: https://doi.org/10.1590/So100-879X2000000500003.

Ruben, R. M., Molinari, M. A., Bibbee, C. A., Childress, M. A., Harman, M. S., Reed, K. P., \& Haff, G. G. (2010). The acute effects of an ascending squat protocol on performance during horizontal plyometric jumps. The Journal of Strength \& Conditioning Research, 24(2), 358-369.Available at: https://doi.org/10.1519/jsc.obo13e3181cc26eo.

Seitz, L. B., de Villarreal, E. S., \& Haff, G. G. (2014). The temporal profile of postactivation potentiation is related to strength level. The Journal of Strength \& Conditioning Research, 28(3), 706-715.Available at: https://doi.org/10.1519/jsc.0bo13e3182a73ea3.

Seitz, L. B., \& Haff, G. G. (2016). Factors modulating post-activation potentiation of jump, sprint, throw, and upper-body ballistic performances: A systematic review with meta-analysis. Sports Medicine, 46(2), 231-240.Available at: https://doi.org/10.1007/s40279-015-0415-7.

Smith, C. E., Hannon, J. C., McGladrey, B., Shultz, B., Eisenman, P., \& Lyons, B. (2014). The effects of a postactivation potentiation warm-up on subsequent sprint performance. Human Movement, 15(1), 36-44.Available at: https://doi.org/10.2478/humo-2013-0050.

Sole, C. J., Moir, G. L., Davis, S. E., \& Witmer, C. A. (2013). Mechanical analysis of the acute effects of a heavy resistance exercise warm-up on agility performance in court-sport athletes. Journal of Human Kinetics, 39(1), 147-156.Available at: https://doi.org/10.2478/hukin-2013-0077.

Stone, M. H., Sands, W. A., Pierce, K. C., Ramsey, M. W., \& Haff, G. G. (2008). Power and power potentiation among strength-power athletes: Preliminary study. International Journal of Sports Physiology and Performance, 3(1), 55-67.Available at: https://doi.org/10.1123/ijspp.3.1.55.

Tillin, N. A., \& Bishop, D. (2009). Factors modulating post-activation potentiation and its effect on performance of subsequent explosive activities. Sports Medicine, 39(2), 147-166.Available at: https://doi.org/10.2165/00007256-200939020-00004.

Turner, A. P., Bellhouse, S., Kilduff, L. P., \& Russell, M. (2015). Postactivation potentiation of sprint acceleration performance using plyometric exercise. The Journal of Strength $\mathcal{E}^{\circ}$ Conditioning Research, 29(2), 343-350.Available at: https://doi.org/10.1519/jsc.0000000000000647.

Whelan, N., O'Regan, C., \& Harrison, A. J. (2014). Resisted sprints do not acutely enhance sprinting performance. The Journal of Strength $\mathbb{E}^{\circ}$ Conditioning Research, 28(7), 1858-1866.Available at: https://doi.org/10.1519/jsc.0000000000000357.

Winwood, P. W., Posthumus, L. R., Cronin, J. B., \& Keogh, J. W. (2016). The acute potentiating effects of heavy sled pulls on sprint performance. The Journal of Strength \& Conditioning Research, 30(5), 1248-1254.Available at: https://doi.org/10.1519/jsc.0000000000001227.

Woolford, S. M., Polglaze, T., Rowsell, G., \& Spencer, M. (2013). Field testing principles and protocols. In: Physiological Tests for Elite Athletes, R. Tanner, C. Gore (Eds.), Australian Institute of Sport (2nd ed., pp. 236). USA: Human Kinetics, IL.

Yilmaz, A. (2019). The acute effect of combined preconditioning strategies on anaerobic performance and physiological parameters in amateur soccer players. Acta Medica Mediterranea, 35(3), 1251-1255.Available at: https://dx.doi.org/10.19193/03936384_2019_3_191. 